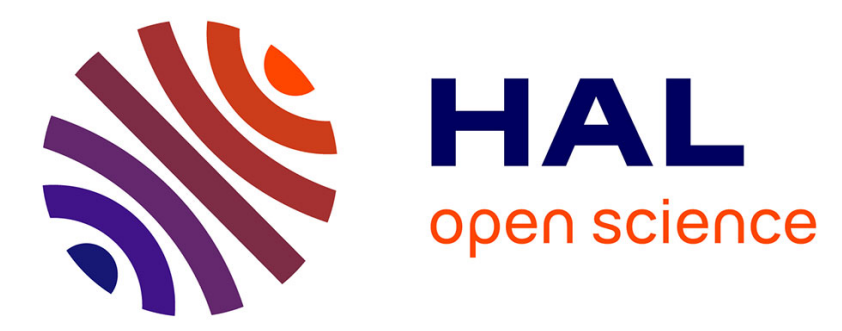

\title{
Selection of optimal cardiac phases for ECG-triggered coronary CT angiography in pediatrics
}

Julien Le Roy, Benoit Azais, Hamid Zarqane, Helene Vernhet Kovacsik, Thibault Mura, Alain Lacampagne, Pascal Amedro

\section{To cite this version:}

Julien Le Roy, Benoit Azais, Hamid Zarqane, Helene Vernhet Kovacsik, Thibault Mura, et al.. Selection of optimal cardiac phases for ECG-triggered coronary CT angiography in pediatrics. Physica Medica, 2021, 81, pp.155-161. 10.1016/j.ejmp.2020.12.002 . hal-03114692

\section{HAL Id: hal-03114692 \\ https://hal.science/hal-03114692}

Submitted on 19 Jan 2021

HAL is a multi-disciplinary open access archive for the deposit and dissemination of scientific research documents, whether they are published or not. The documents may come from teaching and research institutions in France or abroad, or from public or private research centers.
L'archive ouverte pluridisciplinaire HAL, est destinée au dépôt et à la diffusion de documents scientifiques de niveau recherche, publiés ou non, émanant des établissements d'enseignement et de recherche français ou étrangers, des laboratoires publics ou privés. 


\title{
Selection of optimal cardiac phases for ECG-triggered coronary CT angiography in pediatrics
}

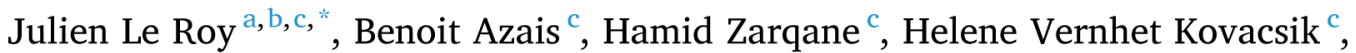 \\ Thibault Mura $^{\mathrm{d}}$, Alain Lacampagne ${ }^{\mathrm{b}}$, Pascal Amedro ${ }^{\mathrm{a}, \mathrm{b}}$ \\ ${ }^{a}$ Pediatric and Congenital Cardiology Department, M3C Regional Reference CHD Center, CHU Montpellier, France \\ ${ }^{\mathrm{b}}$ PhyMedExp, University of Montpellier, CNRS, INSERM, CHU Montpellier, France \\ ${ }^{\mathrm{c}}$ Radiology Department, CHU Montpellier, France \\ ${ }^{\mathrm{d}}$ Epidemiology and Clinical Research Department, University of Montpellier, INSERM, CHU Montpellier, France
}

\section{A R T I C L E I N F O}

\section{Keywords:}

Motion artifacts

Cardiac-gated imaging techniques

Computed tomography angiography

Image quality

\begin{abstract}
A B S T R A C T
Purpose: The use of coronary computed tomography angiography (CCTA) in children remains limited by patient's irradiation, and motion artefacts impairing image quality. Triggering the acquisition at the appropriate moment, and acquiring only necessary components of the cardiac cycle could overcome these limitations. Yet, optimal cardiac intervals to perform CCTA as a function of heart rate (HR) have not yet been addressed in pediatrics. Methods: Fifty children with coronary artery anomalies underwent a CCTA on a wide-coverage single-beat CT scanner. Multiple phases from $25 \%$ to $85 \%$ of the R-R interval were acquired and reconstructed with $10 \%$ increments. Two radiologists independently assessed motion artifacts on each cardiac phase using a 4-point semiquantitative scale.

Results: At patient level, the best phase for acquisition was found in diastole for patients with $\mathrm{HR} \leq 75 \mathrm{bpm}$ and in systole for patients with $\mathrm{HR}>85 \mathrm{bpm}$. At coronary segments and structures level, median optimal phases were reported at $70 \%, 80 \%, 47 \%, 50 \%$, and $54 \%$ of the R-R interval for patients with $\mathrm{HR} \leq 60,61-75,86-100$, 101-130, and $>130 \mathrm{bpm}$ respectively. For patients with HR between 76 and 85 bpm, no clear trend could be observed. Optimal acquisition durations represented 10\% (2 phases), 20\% (3 phases), 50\% (multiphase), 20\% ( 3 phases), and 10\% ( 2 phases) of the R-R interval for patients with $H R \leq 60,61-75,76-100,101-130$, and $>130$ bpm, respectively.

Conclusions: Optimal positioning and duration of CCTA acquisition intervals were investigated as a function of children's HR, to reduce motion artifacts and patient's irradiation.
\end{abstract}

\section{Introduction}

Coronary CT Angiography (CCTA) has been increasingly used in pediatric cardiology over the last decade, and has progressively replaced cardiac catheterization in nearly all situations involving coronary arteries among children with congenital heart diseases (CHD), such as abnormal left or right coronary artery arising from the pulmonary artery, suspected interarterial or intramural coronary artery, coronary artery branch crossing the infundibulum before surgical repair of tetralogy of Fallot, and follow-up after surgical reimplantation of coronary arteries [1]. It is particularly useful to accurately assess cardiac anatomy, when echocardiography or cardiac magnetic resonance might lack of in-depth characterization and spatial resolution, respectively [2]. Cardiac catheterization is a high-performance alternative, but remains an invasive procedure, which delivers significantly higher radiation doses, as compared to CCTA [1].

Despite recent technological improvements, pediatric CCTA remains technically challenging due to the necessity of reducing radiation doses, while providing images of adequate quality for diagnosis. Major technical difficulties include small structures to visualize, fast heart rates, uncontrolled movement or cries, and potential absence of breath hold. Motion artifacts associated with these limitations represent the main

\footnotetext{
Abbreviations: CCTA, coronary computed tomography angiography; HR, heart rate; CHD, congenital heart diseases; Bpm, beats per minute.

* Corresponding author at: Pediatric and Congenital Cardiology Department, Montpellier University Hospital, 371 Avenue du Doyen Giraud, 34295 Montpellier,

E-mail address: j-le_roy@chu-montpellier.fr (J. Le Roy).
} France. 
cause of non-diagnostic CCTA [3].

To overcome these difficulties, several solutions were recently suggested. First, acquisition window's position and duration may be adapted to patient's cardiac characteristics, in order to improve diagnostic confidence while keeping irradiation dose below $1 \mathrm{mSv}$ [4]. Then, second-generation motion correction algorithms could provide higher interpretability rates $(>99 \%)$ in a single cardiac phase, and therefore avoid to acquire most of the cardiac cycle, and decrease irradiation up to $70 \%$ [5]. Finally, despite a certain proportion of non-diagnostic exams, prospective monophasic acquisitions are increasingly used in children, either with dual-source CT helical acquisitions [3], wide-detector CT sequential acquisitions [6], or step and shot technique using single source 64-slices CT [7].

Overall, these solutions require a common prerequisite: a limited interval of the cardiac cycle should be acquired and provide motion-free and interpretable images for diagnosis. Therefore, triggering the acquisition at the right moment within the cardiac cycle and for the right duration is of the utmost importance.

In adult patients, targeting the optimal phase for acquisition is well established, due to moderate and regular heart rates encountered $[8,9]$. However, in pediatrics, disparate cardiac characteristics and very high heart rates might prevent reaching a motionless cardiac phase, when following standard recommendations applied in the adult population. Yet, due to above-mentioned technical challenges of pediatric CCTA, children could highly benefit of an ECG-triggering method adapted to their age.

Consequently, it is of great interest to assess, in a pediatric population, coronary sharpness and motion artifacts at various moments of the cardiac cycle, and establish optimal cardiac moments to trigger CCTA acquisitions as a function of heart rates. Therefore, in this study, we aimed to investigate, in a pediatric population, optimal intervals within the cardiac cycle to trigger CCTA acquisitions.

\section{Materials and methods}

\subsection{Study design and population}

This prospective cross-sectional study was carried out from May 2017 to March 2018 in a tertiary care pediatric and congenital cardiology reference center (Montpellier University Hospital, France). Children with CHD younger than 18 years old, and with a suspected or confirmed coronary artery anomaly, were prospectively recruited. Only children requiring a CCTA as part of their follow-up, and without any allergy to iodinated contrast material, were eligible.

\subsection{Formal aspects}

The study complied with the Good Clinical Practices protocol and Declaration of Helsinki principles. It was approved by the French southeast III Ethics Committee (2017-A00909-44) and registered on ClinicalTrials.gov (NCT03194763).

\subsection{CCTA pediatric protocol}

A prospective ECG-triggered axial technique was performed within a single heart beat for all patients, using a single source, wide-coverage 512-slice CT (Revolution CT, General Electric Healthcare, Milwaukee, USA). Tube voltage ( $\mathrm{kV}$ ) was adapted to patient's weight to maximize iodine contrast to noise ratio: $70 \mathrm{kV}$ for patients weighing less than 12 $\mathrm{kg}, 80 \mathrm{kV}$ between $12 \mathrm{~kg}$ and $35 \mathrm{~kg}$, and $100 \mathrm{kV}$ for more than $35 \mathrm{~kg}$. Automatic tube current modulation (noise index: 24), $0.28 \mathrm{sec}$ gantry rotation time, and $0.625 \mathrm{~mm}$ slice thickness and reconstruction interval were applied. The acquisition started from the aortic arch to the base of the heart and scan ranges were chosen between available lengths of 40 , 80,120 , or $160 \mathrm{~mm}$. The acquisition window was set to acquire cardiac phases from $25 \%$ to $85 \%$ of the R-R interval. Seven reconstructions were performed within this interval, with a $10 \%$ increment. The dose-length product was recorded for every acquisition to evaluate patient exposure.

All patients received an intravenous injection of iodinated contrast medium (Iomeron $400 \mathrm{mg} / \mathrm{ml}$ at $2 \mathrm{ml}$ per $\mathrm{kg}$ intravenously, Bracco Imaging $\mathrm{GmbH}$, Milan, Italy)) [10], followed by $10 \mathrm{ml}$ of saline solution. Injection was manually triggered under the radiologist's supervision at a flow rate ranging from $1.5 \mathrm{ml} / \mathrm{min}$ to $4 \mathrm{ml} / \mathrm{min}$, depending on the patient's age and perfusion capabilities. We used our institutional pediatric CT protocol, as previously reported: one hour before CCTA, eligible children with an HR above 70 beats per minute (bpm) received a single dose of beta-blocker (propranolol $0.5 \mathrm{mg} / \mathrm{kg}$, orally), sedation was prescribed when necessary, using hydroxyzine ( $1 \mathrm{mg} / \mathrm{kg}$, orally) and/or midazolam ( $0.2 \mathrm{mg} / \mathrm{kg}$, orally), upon the pediatric cardiologist's recommendation, without any general anesthesia [4].

\subsection{Image analysis}

The seven reconstructed cardiac phases were assessed independently by two radiologists at cardiac structure level and at patient level. At cardiac structure level, the following structures were evaluated individually: 16 coronary segments [11], left and right coronary ostia, aortic trunk, pulmonary artery, aortic valve, and cardiac chambers. At patient level, the reconstruction was considered as a whole for evaluation.

At both evaluation levels, a four-point semi-quantitative scale derived from previous publication $[5,12]$ was used as follows: excellent result, e.g. minimal or no motion artifacts (4 points); good result, e.g. mild motion artifacts ( 3 points); adequate result, e.g. important artifacts without diagnosis interference ( 2 points); non-diagnostic result, e.g. severe artifacts impairing accurate evaluation (1 point). In case of disagreement, the mean score given by the two observers was finally considered. Only motion artifacts were taken into account: image quality impaired by image noise or injection issue was not taken into account by the radiologists. Heart rates at the time of acquisition were not known by the readers.

Structures with a score $>1$ were considered as interpretable for diagnosis; structures with a score $>2$ were considered as optimal quality for diagnosis; and the proportion of interpretable or optimal structures was defined as the number of structures with a score $>1$ or a score $>2$, respectively, divided by the total number of structures assessed on each cardiac phase.

\subsection{Statistical analysis}

Patient's characteristics were described with proportions for categorical variables and as mean \pm standard deviations (SD) for quantitative variables. At patient level, the phase with the highest score was considered as the best phase. Similarly, a best phase was established for every coronary segment and cardiac structure of each patient. Proportions of interpretable and optimal segments assessed with different number of phases, were performed by retaining the best score of each structure between the different phases gathered. Linear regressions between the position of the best phase and heart rate were performed and correlations were calculated using the Spearman correlation coefficient. Correlation strength was expressed using Evans classification [13]. Agreement of the two independent radiologists for the scoring of motion artifact was assessed using Cohen's Kappa coefficient. Cohen's kappa measures the agreement between two raters beyond the random agreement. According to Landis \& Koch, agreement could be classified as poor $(\mathrm{k}<0.0)$, slight $(\mathrm{k}=0.00-0.20)$, fair $(\mathrm{k}=0.21$ to 0.40$)$, moderate $(\mathrm{k}=0.41-0.60)$, substantial $(\mathrm{k}=0.61-0.80)$, or almost perfect ( $\mathrm{k}$ $=0.80-1.00)[14,15]$. Statistical analyses were performed at a conventional two-tailed $\alpha$ level of 0.05 .

\section{Results}

Fifty pediatric patients ( 24 females, 26 males; $6 \pm 5$ years-old; 
weight: $20 \pm 14$ kg; BMI: $16 \pm 3$ kg.m ${ }^{-2}$; scan length $104 \pm 29 \mathrm{~cm}$ ) were prospectively enrolled in the COROPEDIA clinical trial. All eligible children participated in the study, as no contraindication for CCTA occurred and all families gave their informed consent. All children received a beta-blocker (propranolol $0.5 \mathrm{mg} / \mathrm{kg}$, orally) and sedation with hydroxyzine ( $1 \mathrm{mg} / \mathrm{kg}$, orally). An additional sedation by midazolam $(0.2 \mathrm{mg} / \mathrm{kg}$, orally) was necessary for 25 children, all aged less than 6 years old. No child required underwent general anesthesia. No adverse effect related to beta-blockers, sedation and/or the CCTA itself was reported. Patient mean HR was $95 \pm 26 \mathrm{bpm}$, distributed as follows: $\leq 60 \mathrm{bpm}(\mathrm{N}=6) ; 61-75 \mathrm{bpm}(\mathrm{N}=7) ; 76-85 \mathrm{bpm}(\mathrm{N}=8) ; 86-100 \mathrm{bpm}$ $(\mathrm{N}=10) ; 101-130 \mathrm{bpm}(\mathrm{N}=12)$ and $>130 \mathrm{bpm}(\mathrm{N}=7)$. The median dose length product was 25 mGy.cm (IQR: 16.2-49.1). A total of 5733 cardiac structures were assessed by each radiologist.

At patient level, the best phase for acquisition was found in diastole (from 60 to $85 \%$ of the R-R interval) for patients with $\mathrm{HR} \leq 75 \mathrm{bpm}$, and in systole (from 35 to $60 \%$ of the R-R interval) for patients with HR $>85$ bpm. Among both groups, strong and moderate positive correlations were found between HR and best phase $(r=0.61, P<0.05$ and $r=0.44$, $\mathrm{P}<0.05$, for HR below $75 \mathrm{bpm}$ and above $85 \mathrm{bpm}$, respectively), e.g. the best moment for acquisition shifted towards the end of the R-R interval with increasing HR (Fig. 1). For patients with HR between $76 \mathrm{bpm}$ and $85 \mathrm{bpm}$, no clear trend could be observed (Figs. 1 and 2).

At coronary segment and cardiac structure level, higher proportions of interpretable and optimal structures were reported at $66-75 \%$, $66-85 \%, 36-45 \%$ and $76-85 \%, 36-45 \%, 46-55 \%, 56-65 \%$ of the R-R interval for patient groups with HR of $\leq 60 \mathrm{bpm}, 61-75 \mathrm{bpm}$; 76-85 bpm, 86-100 bpm, 101-130 bpm, and $>130$ bpm, respectively (Figs. 3 and 4 ).

Overall, the proportion of optimal structures increased with a growing number of usable cardiac phases, and declined with increasing HR (Fig. 5A). A similar trend was observed with the proportion of interpretable structures, but no further improvement was observed with additional number of cardiac phases above 3 (Fig. 5B). Considering HR categories, optimal visualization could be obtained with two phases for very low $\mathrm{HR}(\leq 60 \mathrm{bpm})$ and very high $\mathrm{HR}(>130 \mathrm{bpm})$, with three phases for low HR (61-75 bpm) and high HR (101-130 bpm), and with multiple phases, from $35 \%$ to $85 \%$ of the R-R interval, for intermediate HR (76-100 bpm), as reported in Fig. 5 and summarized in Table 1. Regardless of patient's HR, cardiac phases from $25 \%$ to $35 \%$ of the R-R interval did not improve optimal or diagnostic proportion of structures.

Inter-observer agreement was substantial $(\mathrm{k}=0.61$ [0.59-0.62]) for cardiac structure and phase quality assessment.

\section{Discussion}

In a cohort of 50 children with CHD undergoing CCTA, the COROPEDIA trial evaluated cardiac structure sharpness in 7 different cardiac phases, and for 22 different cardiac structures. For the first time, this prospective study assessed optimal cardiac intervals in a pediatric population with wide HR range (from 42 to $185 \mathrm{bpm}$ ), in order to perform ECG-triggered CCTA in children.

\subsection{Optimal position of cardiac phase to acquire in CCTA}

To improve image quality in pediatric CCTA, the most effective strategy is to minimize motion artifacts, and therefore, to trigger the acquisition at the appropriate moment within the cardiac cycle.

In adult populations, two relatively quiescent cardiac phases are considered to trigger CCTA acquisitions: mid-end diastole and end systole. The diastolic stability phase is preferred for patients with low HR but tends to shorten with increasing HR. The systolic stability phase then becomes the longest motionless phase, and is preferred for patients with higher HR. Most studies are in agreement with this general recommendation $[8,9,16,17]$. Yet, the threshold for this transition remains uncertain: HR from 67 to $85 \mathrm{bpm}$ were reported as a limit in adult populations $[8,9,16,18]$, and the unique study performed in children, using MRI, also stated that this threshold localization remained unclear [19]. This topic is of interest, as patients within this HR range are frequent, both in pediatric and adult populations, therefore reaching optimal image quality remains challenging. Our results suggest that a diastolic acquisition should be preferred for children with HR below 75 bpm, while a systolic acquisition should be preferred for HR above 85 $\mathrm{bpm}$, which is in agreement with previously discussed literature results.

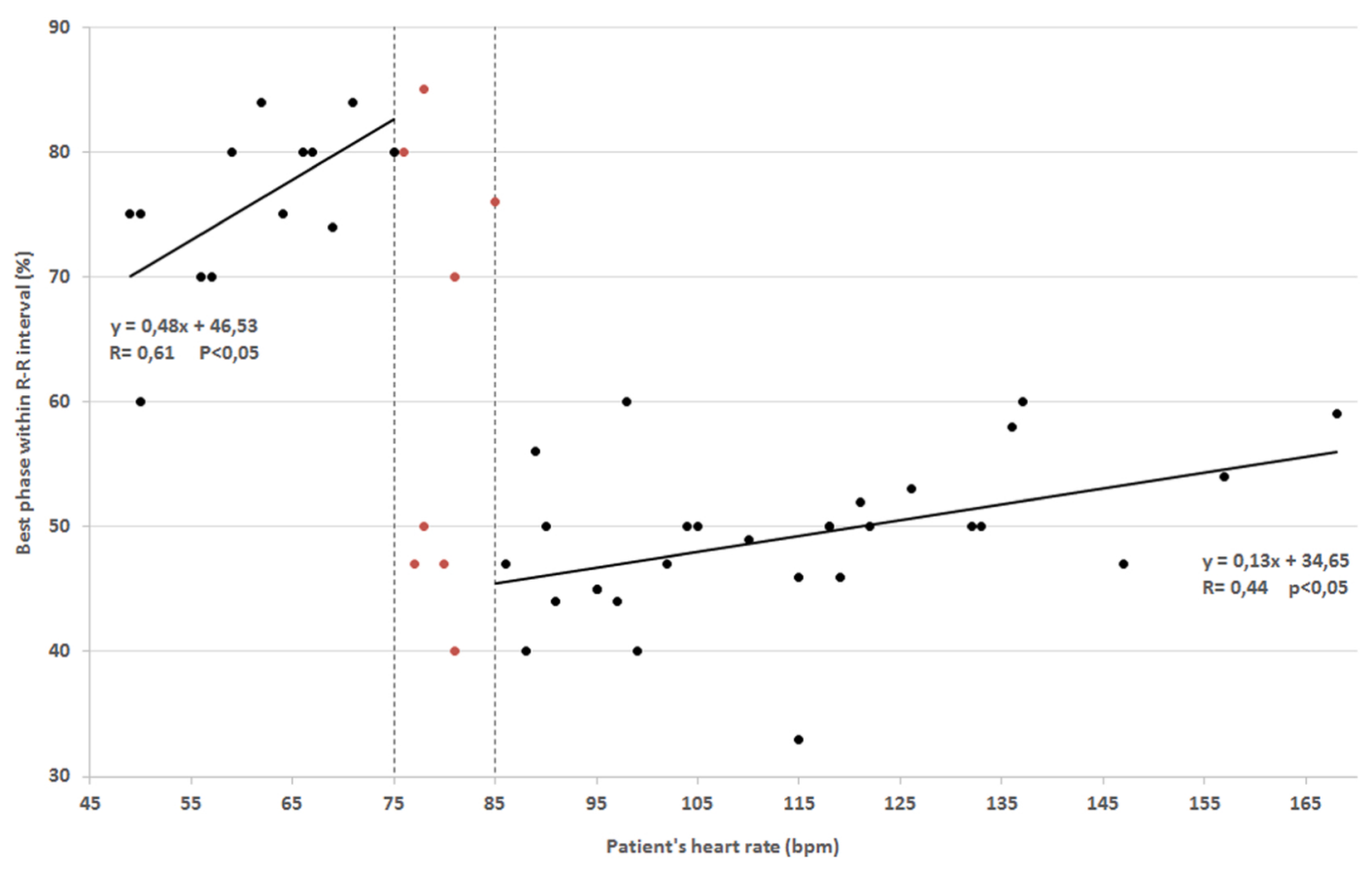

Fig. 1. Position of the best cardiac phase within the R-R interval, at patient level, as a function of patient's heart rate. Diastolic acquisition yielded to best image quality for $\mathrm{HR} \leq 75 \mathrm{bpm}$, while systolic acquisition provided best image quality for $\mathrm{HR}>85 \mathrm{bpm}$. The best moment for acquisition shifted towards the end of the R-R interval with increasing HR for both HR groups $(r=0.61$ and $r=0.44, P<0.05$, respectively). No trend was observed for HR between 75 and 85 bpm. 


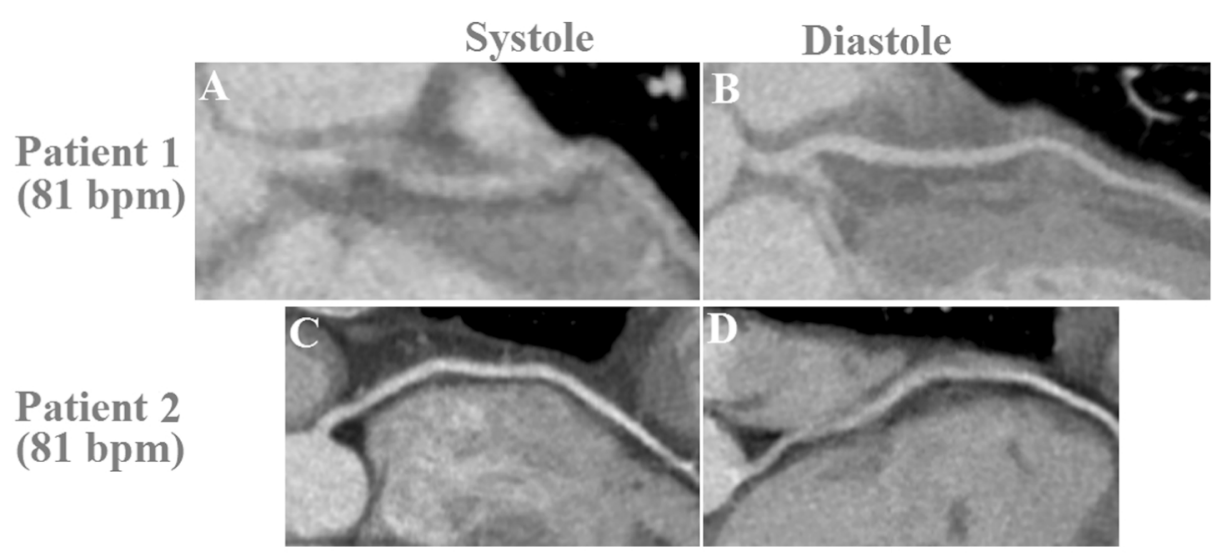

Fig. 2. Curved multiplanar reformations of the right coronary artery performed in two different cardiac phases (systole: A, C; Diastole: B, D) for two children with the exact same heart rate $(81 \mathrm{bpm})$ and a similar heart rate variability. The Optimal cardiac phase was found in diastole for patient 1 (A vs. B) and in systole for patient 2 (C vs. D), illustrating the absence of phase consensus for acquisition in patients with HR between 76 and $85 \mathrm{bpm}$, and the need for multiphasic acquisition in children with intermediate HR.
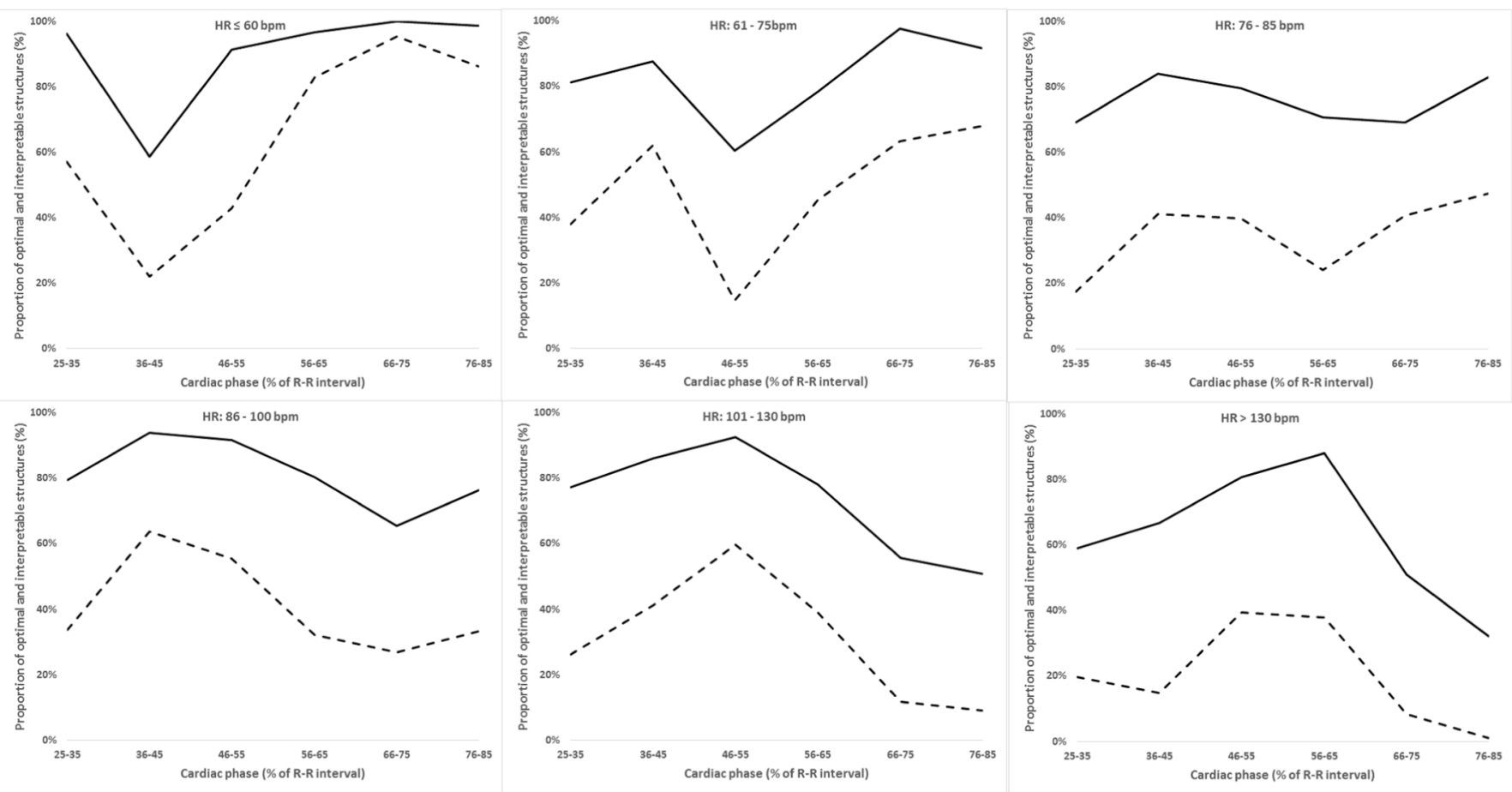

Fig. 3. Evolution of interpretable (solid line) and optimal (dotted line) structure proportions during the cardiac cycle for different heart rate groups. Optimal cardiac intervals were found at 66-75\%, 66-85\%, 36-45\% and 76-85\%, 36-45\%, 46-55\%, 46-65\% of the R-R interval for patients with $\mathrm{HR} \leq 60 \mathrm{bpm}, 61-75 \mathrm{bpm}$, 76-85 bpm, 86-100 bpm, 101-130 bpm, and $>130 \mathrm{bpm}$, respectively. This dynamic assessment provides the most relevant cardiac intervals to prioritize, for multiphasic acquisitions.

No clear trend could be observed regarding patients with HR between 76 and 85 bpm: optimal phases were randomly distributed either in diastole or in systole, even for children with the exact same $\mathrm{HR}$ and similar HR variability (Fig. 2). Similar duration of systolic and diastolic motionless intervals (87 ms vs. $96 \mathrm{~ms}, \mathrm{P}=0.08$ ) [19] at these HR, might have led to inter-patient variability regarding the choice of the phase to acquire. As a consequence, to increase the proportion of interpretable and optimal structures, we suggest to acquire at least one phase during systole and another during diastole for these patients. Heart rate control methods could also be an alternative to reduce phase selection uncertainties and motion artifacts; yet, further studies investigating this intermediate HR range would be necessary.

Additionally, the position of the optimal cardiac phase shifted toward the end of the R-R interval with increasing HR for both systolic and diastolic acquisitions. This has been previously described in adult populations with moderate $\operatorname{HR}[9,20,21]$. Yet, such a shift has not been described at higher HR, to our knowledge [19]. This result is of importance, as ignoring this shift would result in significantly lower interpretability and optimal rates, in particular for HR above $85 \mathrm{bpm}$ (Fig. 3). While duration of most cardiac phases remains fairly consistent, higher HR causes diastasis to shorten [9]. Since acquisitions are triggered following a relative delay from previous R-peak, both diastolic and systolic quiescent intervals shift toward the end of the R-R interval. The use of absolute, or retrograde delays to trigger the acquisition would avoid considering this shift [16].

Finally, the R-R interval duration is influenced by patient's heart rate variability: a mismatch between targeted and acquired phase might happen due to these fluctuations from one beat to another. To mitigate this inherent limitation of prospectively ECG-triggered CCTA, a widen acquisition window might be applied to acquire several cardiac phases.

\subsection{Optimal number of cardiac phases to acquire in CCTA}

To reduce patient radiation exposure, the most effective approach is 

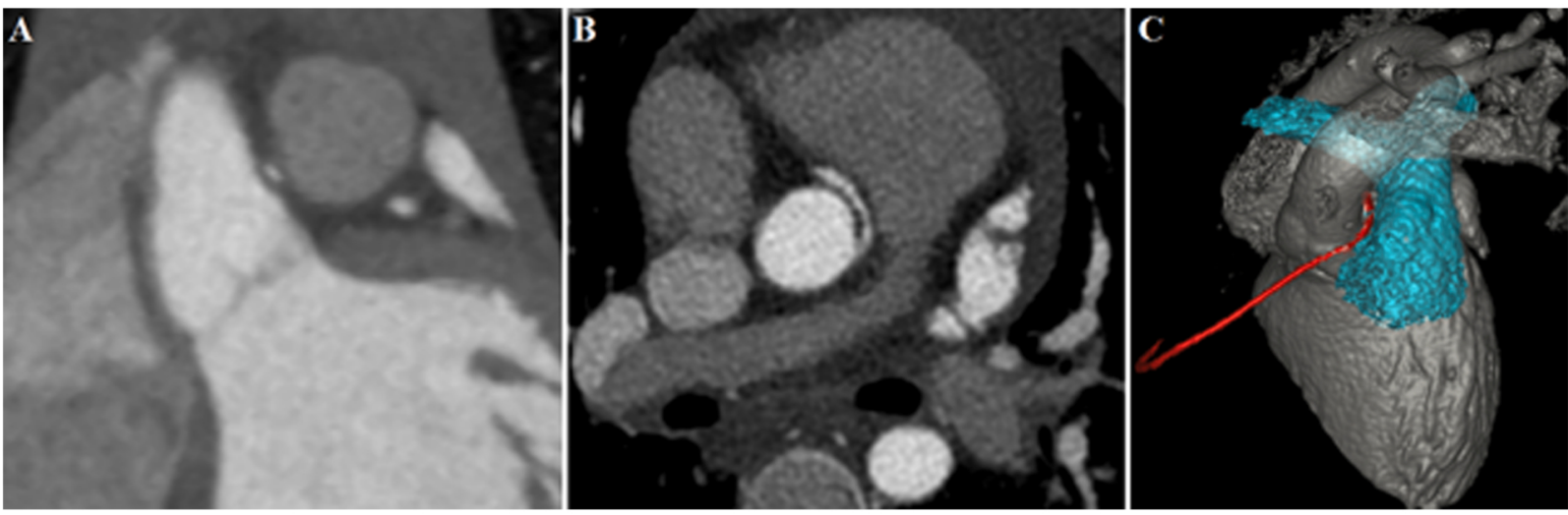

Fig. 4. Coronary computed tomography angiography of a 5-year-old boy (HR $=98 \mathrm{bpm}$ ) with an anomalous origin of the right coronary artery. Sagittal-oblique (A), axial (B) and volume rendered (C) reformations reveal a right coronary artery anomaly, arising from the left ostium. The interaortic pulmonary course induces a stenosis of the proximal portion of the right coronary artery. Highest proportion of diagnostic and optimal structure are observed at $40 \%$ of the R-R interval.
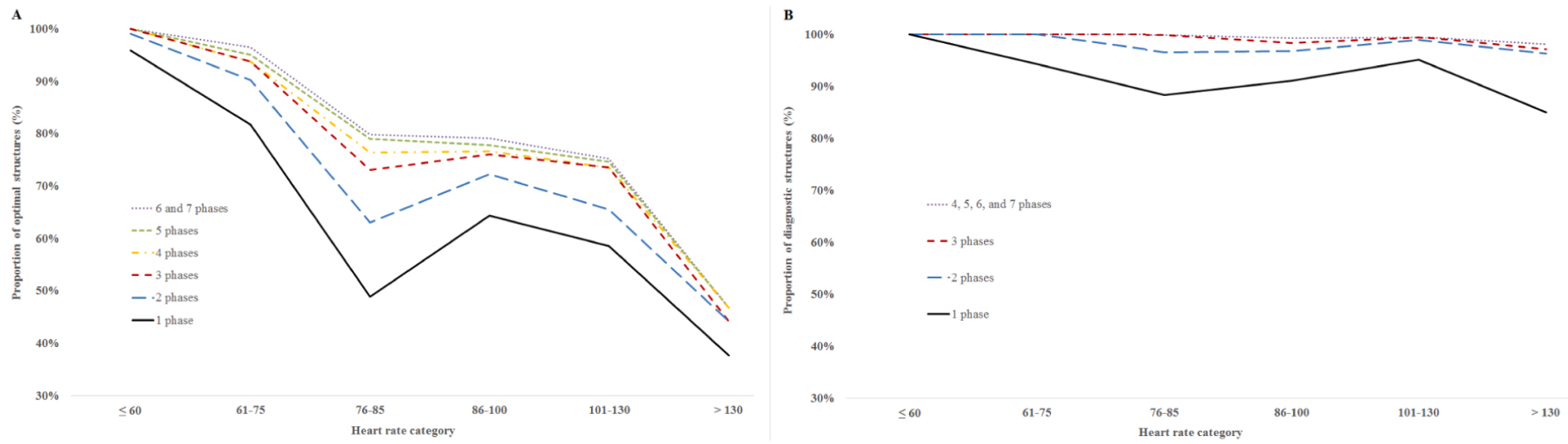

Fig. 5. Proportion of optimal (A) and interpretable (B) structures, when aggregating different numbers of cardiac phases, as a function of patient's heart rate. The proportion of optimal structures increased with the growing number of available cardiac phases, and declined with increasing HR (A). A similar trend was observed with the proportion of interpretable structures, but a consistent interpretability rate was achieved with 3 phases or more (B). Optimal visualization could be obtained with two phases for very low HR ( $<60 \mathrm{bpm})$ and very high HR ( $>130 \mathrm{bpm})$, with three phases for low HR (76-85 bpm) and high HR (101-130 bpm), and with 6 phases for intermediate HR (76-100 bpm).

Table 1

Recommendations for positioning the acquisition window in case of monophasic acquisition, and positioning and duration of acquisition window in case of a multiphasic acquisition.

\begin{tabular}{|c|c|c|c|c|c|c|}
\hline \multirow[t]{2}{*}{ Patient HR (bpm) } & \multicolumn{3}{|c|}{ Cardiac phases (\% of R-R interval) recommended for acquisition } & \multirow[t]{2}{*}{ Leschka [22] Adults CT } & \multirow[t]{2}{*}{ Weustlink [23] Adults CT } & \multirow[t]{2}{*}{ Zhang [19] Children MRI } \\
\hline & Monophasic & Multiphasic & $\mathrm{N}$ phases & & & \\
\hline$\leq 60$ & $70 \%$ & $65-75 \%$ & 2 & $55-70 \%$ & $60-76 \%$ & $73 \%$ \\
\hline $61-75$ & $80 \%$ & $65-85 \%$ & 3 & $50-80 \%$ & $30-77 \%$ & $76 \%$ \\
\hline $76-85$ & $40 \% *$ & $35-85 \%$ & multiple & $45-80 \%$ & & $45 \%$ and $85 \%$ \\
\hline $86-100$ & $47 \%$ & $35-85 \%$ & multiple & $30-80 \%$ & $31-47 \%$ & $48 \%$ \\
\hline $101-130$ & $50 \%$ & $40-60 \%$ & 3 & & & $50-55 \%$ \\
\hline$>130$ & $54 \%$ & $50-60 \%$ & 2 & & & $55 \%$ \\
\hline
\end{tabular}

Legend: HR, heart rate.

*Best score was found at $40 \%$ of the R-R interval in patients with HR between $76 \mathrm{bpm}$ and $85 \mathrm{bpm}$. Yet, a second acquisition performed in diastole is highly recommended due to uncertainties regarding best cardiac phase encountered at these HR.

to limit beam on time and therefore acquire only necessary parts of the cardiac cycle. Our results suggest that the number of phases acquired should be adapted to children HR: intermediate HR (80-100 bpm) require multiple cardiac phases while, as HR progresses to extreme values (both to higher and lower end of pediatric HR ranges), the number of cardiac phases necessary to provide optimal exams is reduced to three, and then to two phases (representing respectively $20 \%$ and $10 \%$ of the cardiac cycle). To our knowledge, no study previously addressed the number of cardiac phases required in pediatric CCTA, and very few data is available in adult population.
Overall, our results are in line with those from Lescha et al., who established narrowest reconstruction windows as a function of HR in adults [22]. As in our study, the authors recommended to use $10 \%$ to $20 \%$ of R-R interval at end diastole for patients with HR below $60 \mathrm{bpm}$ and between $60 \mathrm{bpm}$ and $70 \mathrm{bpm}$ respectively, while a wider multiphasic approach was recommended for patients with HR above $70 \mathrm{bpm}$. Similarly to our results, Weustink et al. recommended a narrow acquisition window for patients with low HR and high HR, while a wider window was necessary for intermediate HR.[23] Yet, these studies did not specifically address the number of cardiac phases necessary for HR 
above $100 \mathrm{bpm}$, as commonly encountered in pediatrics. Surprisingly, in our study, the acquisition of two to three phases represented the best compromise, therefore much less than for intermediate HR.

Our conclusions are supported by the recent study from Zang et al., which measured the duration of motionless cardiac intervals in children, as a function of HR, using magnetic resonance imaging [19]. At lower and higher end of pediatric HR ranges, significant differences between the duration of quiescent systolic and diastolic intervals were reported ( $80 \mathrm{~ms}$ vs. $335 \mathrm{~ms}, 72 \mathrm{~ms}$ vs. $212 \mathrm{~ms}, 77 \mathrm{~ms}$ vs. $131 \mathrm{~ms}, 105 \mathrm{~ms}$ vs. $61 \mathrm{~ms}$, $106 \mathrm{~ms}$ vs. $49 \mathrm{~ms}, 105 \mathrm{~ms}$ vs. $38 \mathrm{~ms}, 97 \mathrm{~ms}$ vs. $38 \mathrm{~ms}$, for systolic vs. diastolic static durations for, respectively, HR $<60 \mathrm{bpm}, 60-69 \mathrm{bpm}$, 70-79 bpm, 100-109 bpm, 110-119 bpm, 120-129 bpm and > 130 bpm, all $\mathrm{P}<0.0001$ ). As a consequence, acquiring well-defined structures in other cardiac phase than in systole (for high HR), or than in diastole (for low HR), appears very unlikely, and a small number of cardiac phases positioned during the longer quiescent phase seems adequate to reach optimal image quality. On the opposite, similar durations of diastolic and systolic quiescent intervals were reported in patients with HR between 80 bpm and 99 bpm (87 ms vs. 71-96 ms, P > 0.01 ), increasing probabilities of finding sharper structures in another cardiac phase, as previously described [4], which strengthens the need for multiphasic acquisitions at such HR range.

\subsection{Limitations}

Given radiation safety considerations, it was not possible to acquire the entire cardiac cycle at full dose in a pediatric cohort. Therefore, some cardiac phases were acquired with a reduced current value. This may have artificially increased the proportion of poorly visualized structures in certain cardiac phases. However, we made sure to only assess structure sharpness related to motion; indeed, other discrepancies, such as noise, were not considered in the assessment. Moreover, a minimum irradiation time is necessary to complete any CT acquisition ( $140 \mathrm{~ms}$ in this work). This device-specific duration was not considered in our study. Yet, this absolute time may represent an important portion, relative to shorter $\mathrm{R}-\mathrm{R}$ intervals at higher $\mathrm{HR}$, forcing longer acquisitions than recommended this work. Additionally, our results might not entirely be applicable to CT systems with very different temporal resolutions, involving various capabilities to freeze coronary artery movement. In particular dual-source CT, a vendor specific technology provided by one manufacturer, might require fewer phases than recommended in this study. Yet, the authors believe that these results can still serve as a basis for CCTA optimization on these systems, since no similar study with any other equipment is available in pediatrics to date to our knowledge.

\subsection{Conclusions}

The COROPEDIA trial investigated optimal cardiac intervals to perform pediatric CCTA acquisitions. Optimal positioning and duration of the acquisition window were determined, as a function of children's HR. Diastole should be preferred for HR $\leq 75 \mathrm{bpm}$, systole for HR $>85$ bpm and the number of acquired cardiac phases should be increased for intermediate $\mathrm{HR}$, especially in the $75-85 \mathrm{bpm}$ range, where a single cardiac phase appears uncertain to provide adequate image quality. Yet, additional studies would be necessary in this particular HR range. These recommendations should be applied on prospectively ECG-triggered CCTA, in order to reduce motion artifacts and improve image quality, while reducing patient's radiation exposure and, in the long term, associated radiation-induced cancers.

\section{References}

[1] Yang J-T, Lin M-T, Jaw F-S, Chen S-J, Wang J-K, Shih T-F, Wu M-H, Li Y-W. Trends in the utilization of computed tomography and cardiac catheterization among children with congenital heart disease. J Formos Med Assoc 2015;114(11):1061-8. https://doi.org/10.1016/j.jfma.2014.08.004.

[2] Goitein O, Salem Y, Jacobson J, Goitein D, Mishali D, Hamdan A, et al. The role of cardiac computed tomography in infants with congenital heart disease. Isr Med Assoc J. 2014;16:147-52.

[3] Liu Y, Li J, Zhao H, Jia Y, Ren J, Xu J, Hao Y, Zheng M. Image quality and radiation dose of dual-source CT cardiac angiography using prospective ECG-triggering technique in pediatric patients with congenital heart disease. J Cardiothorac Surg 2016;11(1). https://doi.org/10.1186/s13019-016-0460-9.

[4] Le Roy J, Vernhet Kovacsik H, Zarqane H, Vincenti M, Abassi H, Lavastre K, Mura T, Lacampagne A, Amedro P. Submillisievert multiphasic coronary computed tomography angiography for pediatric patients with congenital heart diseases. Circ: Cardiovascular Imaging 2019;12(2). https://doi.org/10.1161/ CIRCIMAGING.118.008348.

[5] Le Roy J, Zarqane H, Azais B, Vernhet Kovacsik H, Mura T, Okerlund D, Lacampagne A, Amedro P. Impact of motion correction algorithms on image quality in children undergoing coronary computed tomography angiography: a comparison with regular monophasic and multiphasic acquisitions. Circ: Cardiovascular Imaging 2019;12(12). https://doi.org/10.1161/ CIRCIMAGING.119.009650.

[6] Jadhav SP, Golriz F, Atweh LA, Zhang W, Krishnamurthy R. CT angiography of neonates and infants: comparison of radiation dose and image quality of target mode prospectively ECG-Gated 320-MDCT and ungated helical 64-MDCT. Am J Roentgenol 2015;204(2):W184-91. https://doi.org/10.2214/AJR.14.12846.

[7] Habib Geryes B, Calmon R, Donciu V, Khraiche D, Warin-Fresse K, Bonnet D, Boddaert N, Raimondi F. Low-dose paediatric cardiac and thoracic computed tomography with prospective triggering: Is it possible at any heart rate? Physica Med 2018;49:99-104. https://doi.org/10.1016/j.ejmp.2018.05.015.

[8] Araoz PA, Kirsch J, Primak AN, Braun NN, Saba O, Williamson EE, Harmsen WS, Mandrekar JN, McCollough CH. Optimal image reconstruction phase at low and high heart rates in dual-source CT coronary angiography. Int J Cardiovase Imaging 2009;25(8):837-45. https://doi.org/10.1007/s10554-009-9489-3.

[9] Seifarth H, Wienbeck S, Püsken M, Juergens K-U, Maintz D, Vahlhaus C, Heindel W, Fischbach R. Optimal systolic and diastolic reconstruction windows for coronary CT angiography using dual-source CT. Am J Roentgenol 2007;189(6): 1317-23. https://doi.org/10.2214/AJR.07.2711.

[10] Becker CR, Vanzulli A, Fink C, de Faveri D, Fedeli S, Dore R, Biondetti P, Kuettner A, Krix M, Ascenti G. Multicenter comparison of high concentration contrast agent iomeprol-400 with iso-osmolar Iodixanol-320: contrast enhancement and heart rate variation in coronary dual-source computed tomographic angiography. Invest Radiol 2011;46(7):457-64. https://doi.org/ 10.1097/RLI.0b013e31821c7ff4.

[11] Austen WG, Edwards JE, Frye RL, Gensini GG, Gott VL, Griffith LS, McGoon DC, Murphy ML, Roe BB. A reporting system on patients evaluated for coronary artery disease. Report of the Ad Hoc Committee for Grading of Coronary Artery Disease, Council on Cardiovascular Surgery, American Heart Association. Circulation 1975; 51(4):5-40. https://doi.org/10.1161/01.CIR.51.4.5.

[12] Andreini D, Pontone G, Mushtaq S, Bertella E, Conte E, Segurini C, Baggiano A, Bartorelli AL, Annoni A, Formenti A, Petullà M, Beltrama V, Fiorentini C, Pepi M. Low-dose CT coronary angiography with a novel IntraCycle motion-correction algorithm in patients with high heart rate or heart rate variability. Eur Heart $\mathbf{J}$ Cardiovasc Imaging 2015;16(10):1093-100. https://doi.org/10.1093/ehjci/ jev033.

[13] Evans JD. Straightforward statistics for the behavioral sciences. Pacific Grove: Brooks/Cole Publishing; 1996.

[14] Landis JR, Koch GG. The measurement of observer agreement for categorical data. Biometrics 1977;33(1):159. https://doi.org/10.2307/2529310.

[15] McHugh ML. Interrater reliability: the kappa statistic. Biochem Med 2012:276-82. https://doi.org/10.11613/BM.2012.031.

[16] Herzog C, Abolmaali N, Balzer JO, Baunach S, Ackermann H, Dogan S, Britten MB, Vogl TJ. Heart-rate-adapted image reconstruction in multidetector-row cardiac CT: influence of physiological and technical prerequisite on image quality. Eur Radiol 2002;12(11):2670-8. https://doi.org/10.1007/s00330-002-1553-5.

[17] Husmann L, Leschka S, Desbiolles L, Schepis T, Gaemperli O, Seifert B, Cattin P, Frauenfelder T, Flohr TG, Marincek B, Kaufmann PA, Alkadhi H. Coronary artery motion and cardiac phases: dependency on heart rate-implications for CT image reconstruction. Radiology 2007;245(2):567-76. https://doi.org/10.1148/ radiol.2451061791.

[18] Leschka S, Wildermuth S, Boehm T, Desbiolles L, Husmann L, Plass A, Koepfli P, Schepis T, Marincek B, Kaufmann PA, Alkadhi H. Noninvasive coronary angiography with 64-section CT: effect of average heart rate and heart rate variability on image quality. Radiology 2006;241(2):378-85. https://doi.org/ 10.1148/radiol.2412051384.

[19] Zhang W, Bogale S, Golriz F, Krishnamurthy R. Relationship between heart rate and quiescent interval of the cardiac cycle in children using MRI. Pediatr Radiol 2017;47(12):1588-93. https://doi.org/10.1007/s00247-017-3918-6.

[20] Kim WY, Stuber M, Kissinger KV, Andersen NT, Manning WJ, Botnar RM. Impact of bulk cardiac motion on right coronary MR angiography and vessel wall imaging. J. Magn. Reson. Imaging 2001;14(4):383-90. https://doi.org/10.1002/jmri.1198.

[21] Hoffmann MHK, Shi H, Manzke R, Schmid FT, De Vries L, Grass M, Brambs H-J, Aschoff AJ. Noninvasive coronary angiography with 16-detector row CT: effect of 
heart rate. Radiology 2005;234(1):86-97. https://doi oro/10.1148/ radiol.2341031408.

[22] Leschka S, Scheffel H, Desbiolles L, Plass A, Gaemperli O, Valenta I, Husmann L Flohr TG, Genoni M, Marincek B, Kaufmann PA, Alkadhi H. Image quality and reconstruction intervals of dual-source $\mathrm{CT}$ coronary angiography:

recommendations for ECG-pulsing windowing. Invest Radiol 2007;42(8):543-9. https://doi.org/10.1097/RLI.0b013e31803b93cf.
[23] Weustink AC, Mollet NR, Pugliese F, Meijboom WB, Nieman K, Heijenbrok-Kal MH, Flohr TG, Neefjes LAE, Cademartiri F, de Feyter PJ, Krestin GP. Optimal electrocardiographic pulsing windows and heart rate: effect on image quality and radiation exposure at dual-source coronary CT angiography. Radiology 2008;248 (3):792-8. https://doi.org/10.1148/radiol.2483072098. 